\title{
Ergonomic evaluation of postural assessment among "canting" batik workers
}

\author{
Norliana Yusof ${ }^{1}$, Rozimah Yusof ${ }^{2}$, Firoz @ Mohd Firoz Ahmat Basri ${ }^{3}$, \\ Norhayati Soin ${ }^{4}$ \\ ${ }^{1,2,3}$ Faculty of Innovative Design and Technology, Universiti Sultan Zainal Abidin, Kampus \\ Gong Badak, 21300 Kuala Terengganu, Terengganu, Malaysia \\ ${ }^{4}$ Department of Electrical Engineering, Faculty of Engineering, University of Malaya, 50603 \\ Kuala Lumpur, Malaysia
}

1norliana@unisza.edu.my, ${ }^{2}$ rozimah@unisza.edu.my, ${ }^{3}$ firoz@unisza.edu.my,
nnorhayatisoin@um.edu.my

Keywords : batik, posture, musculoskeletal disorders, RULA,REBA

\begin{abstract}
This paper presents a study in batik industry with the objective of determining any musculoskeletal disorders (MSDs) among "canting" batik workers in Kuala Terengganu. The MSDs symptoms were identified through questionnaires modified from a Standardized Nordic Questionnaire (SNQ) and from observational techniques by using a postural analysis tool, Rapid Upper Limb Assessment (RULA) and Rapid Entire Body Assessment (REBA). The results of questionnaires revealed that MSDs occurred at a high rate which the highest rates of MSDs prevalence were reported in shoulders (64.5\%). By assessing postural using the REBA software, the score 4 placed it in medium risk level. However, there are other factors contribute to the existence of MSD such as prolong standing, repetitive movement and poor workstation design. These parameters are not taken into account in the RULA and REBA analysis. Thus, designing an ergonomic workstation may prevent awkward postures to provide a maximum comfort to the batik workers and consequently enhance the productivity.
\end{abstract}

\section{INTRODUCTION}

Batik industry is one of the oldest industries in Malaysia. The industry of batik in Malaysia has been existed around the year of 1921. Throughout the years of its existence, the industry contributes to the Malaysian economic well being by first, creating jobs and business opportunities in rural areas [1]. In Malaysia, there are two major types of batik processes, which are Hand-drawn and Block-printed batik. Hand-drawn batik is where designs are drawn on fabric or cloth with hot liquid wax by using a metal object called "canting". When the wax outlines are done, the workers use the brushes to paint the dyes within the outlines. The use of brush allows for the creation of shaded and multi-hued designs. Prior to the "canting" process, this cloth is stretched across two meter length frames with at least one waist height. The cloth is tacked periodically to keep the material taut. The workers will do the "canting" process in standing mode differently from Javanese, where "canting" workers do the process by sitting down on the floor. Most of the handdrawn, canting batik artists or workers are young women whereas for block-printed batik is mostly dominated by men [2].

Several physical risk factors for work-related musculoskeletal disorders (WMSDs) can be identified in working life such as postures, manual handling high peak load, static load, vibration, repetitive work, contact stress, speed or acceleration of movement $[3,4]$ There are many researchers 
studied on the discomfort working postures by using different method. According to Razlan et al. [5] in their study on batik workers in Kelantan more than half $(60.2 \%)$ of the workers had been troubled with musculoskeletal systems at work. The risk of musculoskeletal injury associated with the recorded posture(s), in the context of a full ergonomic workplace assessment, can be a major factor for implementing changes [6]. Most postural analysis techniques have two, usually contradictory, qualities of generality and sensitivity [6,7] REBA has been developed as a postural analysis system sensitive to musculoskeletal risks in a variety of tasks. Although the REBA promises as a useful postural analysis tool, further validation needs to be carried out [6].

\section{METHODOLOGY}

\subsection{Questionnaire, Interview and Observation.}

In this survey, 41 batik workers were randomly selected and those with background of having diseases or accidents affecting musculoskeletal system were excluded from the study. The questionnaires consisted of two parts covering personal details and musculoskeletal problems in different body regions. Upon completion of the fields study, the data was analyzed by using SPSS version 17 software.

The President of the Persatuan Pembatik Terengganu, Puan Wan Noordiran Wan Long, was also interviewed to know the present situation of the batik industry in Terengganu. Observation of canting process was also done at two batik workshops.

\subsection{RULA and REBA analysis}

In conducting postural assessment using RULA and REBA, a video and still pictures as in Figure 5 of a worker doing her job activity were analyzed. According to REBA method, a score is calculated for the posture of each body part. Score 1 indicates the most neutral posture and score 13 shows the worst position. A REBA score of 1-3 is considered acceptable; scores of 4-7 indicate a medium risk with necessary action. A score 8-10 indicates high risk with action soon, and a score 11-15 indicates very high risk with immediate action [6]. Based on RULA scoring system, four action levels were suggested [8]. Action level 1 :Posture is acceptable, Action level 2: Further investigation is needed and changes may be needed, Action level 3 :Investigation and changes are required soon and Action level 4: Investigation and changes are required immediately.

\section{RESULTS}

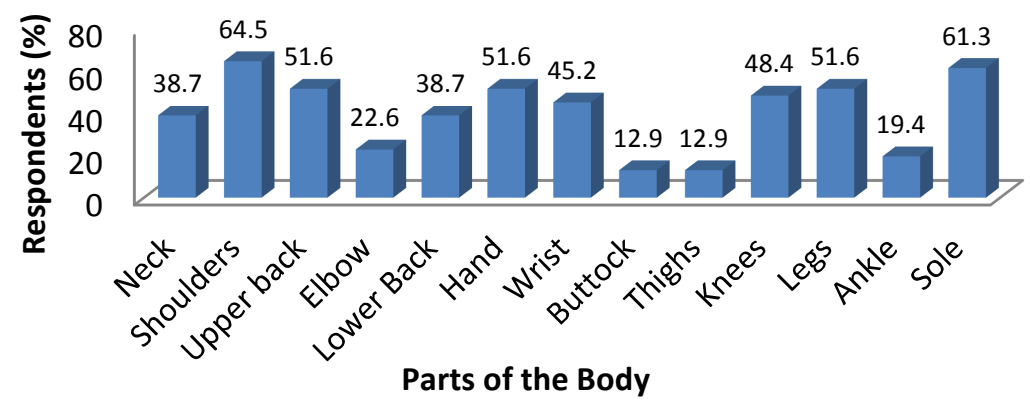

Fig. 1. MSDs Complaints

Figure 1 shows the prevalence of MSDs symptoms complained by the respondents. The most commonly affected regions among batik workers were shoulders $(64.5 \%)$, sole $(61.3 \%)$, upper back (51.6\%), hand (51.6\%), legs (51.6\%), knee (48.4\%) and wrist (45.2\%). 
Figure 2 shows that working hours of more than 6 hours per day also contributes to MSDs complaints. From the observation, most of the workers did not have proper rest after each "canting" process. Shoulder and sole pain complaints are most probably due to the prolonged standing hours.

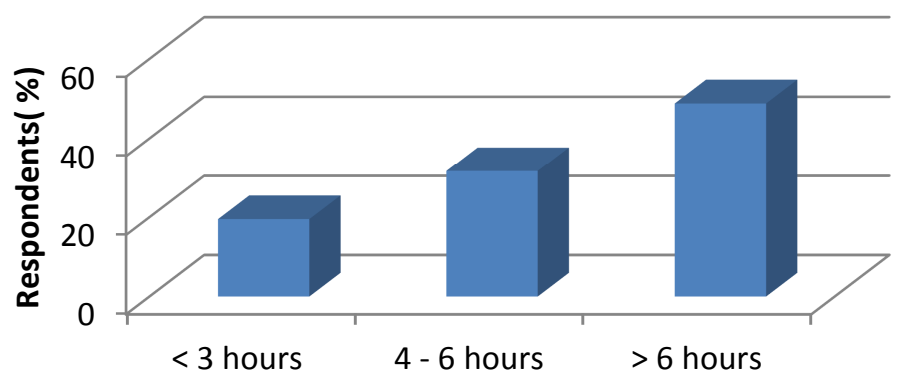

Fig. 2. Working Hours

Figure 3 shows the RULA Grand Score for right hand side was 6 and for left hand side were 4. A score of 5 or 6 indicates that the level of exposure to MSDs risks was high and ergonomics intervention to decrease the symptoms seemed essential (action level 3). A score of between 3 and 4 indicates that the level of exposure needed considering (action level 2).

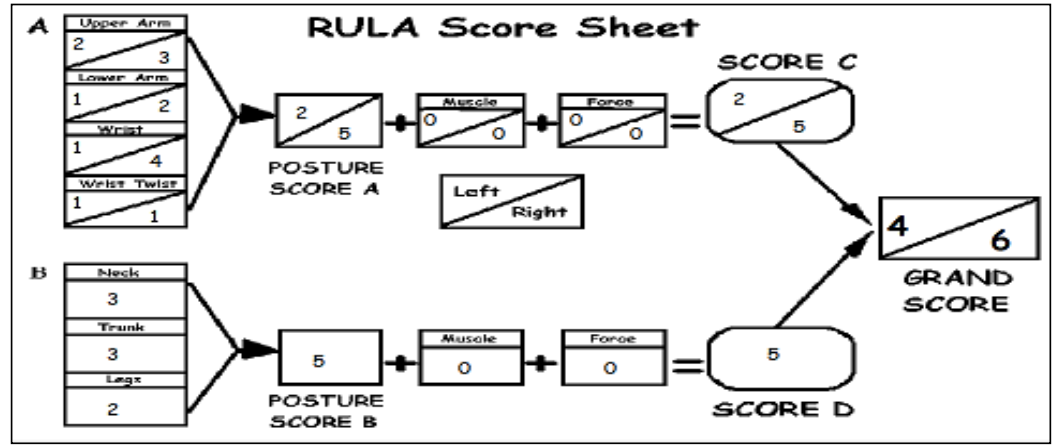

Fig.3. RULA score

Figure 4 shows REBA analysis had a grand score of 9 for right hand side and 7 for left hand side. The total score indicates a high prevalence symptom of MSDs among the "canting" batik workers for the right hand side and at a medium risk for the left hand side

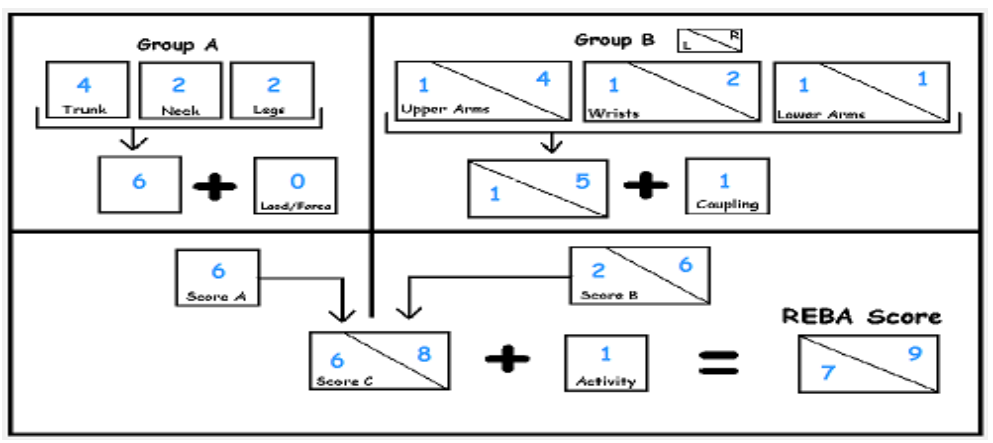

Fig.4. REBA score

\section{DISCUSSIONS}

Our study showed that $77.8 \%$ of the respondents suffered from at least one work related musculoskeletal disorders in the last 12 months. This is in agreement with study by Razlan et al. [5] which reported that $60.2 \%$ of the batik workers had musculoskeletal problems at work. From our 
survey, the most commonly affected regions of the subjects are shoulder and sole. From the RULA and REBA analysis, both indicates that exposure to MSDs for the right hand side is high since the "canting" process only actively involves one hand. This could be due to the body flexion of trunk at between angle $20-60^{\circ}$ and the neck flexion at angle $20-40^{\circ}$. Figure 5 shows a batik worker bent her body to "canting" on the upper part of the cloth. Sometimes she had to tip toe to reach at the other side of the cloth.

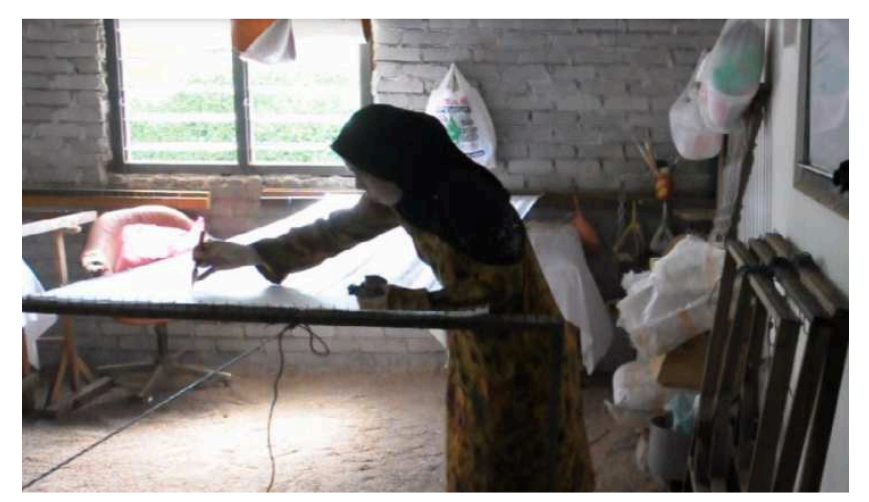

Fig.5. A "canting" process

The second highest commonly affected region is the sole. This is due to the prolonged standing and repetitive movement. From our survey, the respondents of this study were standing for at least six hours per day in order to perform their task. Repetitive movement is one of other factors which contributes to the sole pain. From the video recorded, the worker had to walk to the wax pot located almost two meters away from the "canting" table and refilled the wax for every minute. The poor design of "canting" workstation and tools are believed contributing to the factors of repetitive movement in the "canting" process. From the interview with the President of Pembatik in Terengganu, Puan Wan Noordiran Wan Long, it was found out that the Batik entrepreneurs had difficulty in employing "canting" workers and retaining them as their employees. Most of the workers can only last up to 3-4 years and stopped working due to many factors. From our study, it is believed that the factors influenced the scenario include the MSDs prevalence among them and socio economics factor.

\section{CONCLUSION}

This study highlighted the high prevalence of musculoskeletal disorders symptoms among "canting" batik workers. Implications for the batik workers with the high prevalence of MSDs include pain, discomfort, injury, lost productivity and intangible effects on lifestyle which may result in retention problems for this job. The high prevalence of MSDs justifies for further research in prospective task analysis, postural analysis or workstation layout design. In this study, no attempt was made to measure the effects of the developed "canting" workstation on batik workers' performance. Further field trials will be needed to test efficiency under real production conditions. 


\section{REFERENCES}

[1] Wan Hashim W. T. 1996, Malay Handicraft: Industries Origins and Development, Dewan Bahasa Pustaka, Kuala Lumpur

[2] Hanipah Hussin, Ruziah Ali and Ahmad Yusairi Bani Hashim, 2011 "Batik: Lambang Warisan Teknologi dan Inovasi”, Melaka, Malaysia

[3] A.R. Ismail , M.L.Yeo, M.H.M.Haniff, R.Zulkifli, B.M. Deros,and N.K.Makhtar , 2009, Assessment of Postural Loading among the Assembly Operators: A Case Study at Malaysian Automotive Industry, European Journal of Scientific Research, Vol. 30, No 2, pp 224-235

[4] S.Pinzke, L.Kopp. 2001, Marker-less systems for tracking working postures: results from two experiments. Applied Ergonomics, 32: 461-471

[5] RazlanMusa,WinKyi and Rampal K.G., 2000 "Work-Related Musculoskeletal Symptoms Among Batik Workers In Kelantan", Malaysian Journal of Medical Sciences, Vol 7.No.2,July 2000(13-17)

[6] Hignett, S. and Mc Atamney,L., 2000, Rapid Entire Body Assessment (REBA), Applied Ergonomics 31: 201-205

[7] Fransson-Hall, C.,Gloria,R.,Kilbom,A.,Winkel,J.,1995, A Portable Ergonomic Observation Method (POE) for computerized on-line recording of postures and manual handling. Applied Ergonomics 26(2): 93-100

[8] Mc Attaney,L.,Corlett,E.N., 1993.RULA: A Survey Method for the Investigation of Work Related Upper Limb Disorders. Applied Ergonomics 24(2): 91 - 92 